\title{
Target Coil Embolization Using the Combined Transarterial and Transvenous Balloon-assisted Technique for Traumatic Direct Carotid Cavernous Fistula
}

\author{
Ryotaro SuZUKi, ${ }^{1}$ Tomoji TAKIGAwA, ${ }^{1}$ Yoshiyuki MatsumOTO, ${ }^{1}$ Yoshiko FujII, ${ }^{1}$ \\ Yasuhiko NARIAI, ${ }^{1}$ Yoshiki SugIURA, ${ }^{1}$ Yosuke KAWAMURA, ${ }^{1}$ Issei TAKANO, ${ }^{1}$ \\ Yoshihiro TANAKA, ${ }^{1}$ Masaya NAGAISHI, ${ }^{1}$ Akio HYODO, ${ }^{1}$ and Kensuke SUZUKI ${ }^{1}$ \\ ${ }^{1}$ Department of Neurosurgery, Dokkyo Medical University Saitama Medical Center,
} Koshigaya, Saitama, Japan

\begin{abstract}
Herein, we describe a case of traumatic direct carotid cavernous fistula (DCCF) treated with target coil embolization using the combined transarterial and transvenous balloon-assisted technique. The patient was a 59-year-old woman who had been involved in a vehicular accident. She was admitted to the hospital due to chemosis and exophthalmos. Cerebral angiography revealed a shunt from the internal carotid artery (ICA) to the cavernous sinus (CS), which indicated DCCF. Thus, target coil embolization using the combined transarterial and transvenous balloon-assisted technique was performed. Angiography was performed 1 week after surgery to confirm the disappearance of DCCF. No recurrence was observed during the 1-year follow-up after treatment. Thus, target coil embolization using the combined transarterial and transvenous balloon-assisted technique is safe and effective for the treatment of traumatic DCCF.
\end{abstract}

Keywords: direct carotid cavernous fistula, combined transarterial and transvenous balloon-assisted technique, target coil embolization

\section{Introduction}

Endovascular therapy is the first-line treatment for direct carotid cavernous fistula (DCCF), and various endovascular therapies are used for the management of this condition. Transarterial balloon-assisted coil embolization has been used for the treatment of DCCF, ${ }^{1-6)}$ but not transvenous balloon-assisted coil embolization. The transvenous balloon-assisted technique has only been utilized for the treatment of dural arteriovenous fistula (DAVF) using Onyx or N-butyl-2-cyanoacrylate (NBCA) for flow control and prevention of migration of embolic materials to the adjacent blood vessels. ${ }^{7-11)}$ In all patients with such conditions in previous studies, balloon-assisted embolization in the transverse sinus ${ }^{7-9)}$ or superior

Received February 18, 2020; Accepted April 9, 2020

Copyright $\subseteq 2021$ by The Japan Neurosurgical Society This work is licensed under a Creative Commons AttributionNonCommercial-NoDerivatives International License. sagittal sinus (SSS) ${ }^{10,11)}$ was performed, except in one case that underwent balloon-assisted embolization in the cavernous sinus (CS).

If the shunt point is identified, target embolization prevents cranial neuropathy caused by CS packing, and fewer coils are also required in these cases. Herein, we describe successful treatment of a patient with traumatic DCCF with target coil embolization using the combined transarterial and transvenous balloon-assisted technique. Moreover, the key points and efficacy of this technique were discussed.

\section{Case Report}

The patient was a 59-year-old woman who hit her head after falling while riding a bicycle. The patient visited a local physician due to tinnitus, exophthalmos and bruit, and bulbar conjunctival hyperemia in her right eye that developed immediately, approximately 3 months, and 4 months after the accident, respectively. Magnetic resonance imaging (MRI) revealed dilation of the superior ophthalmic vein 

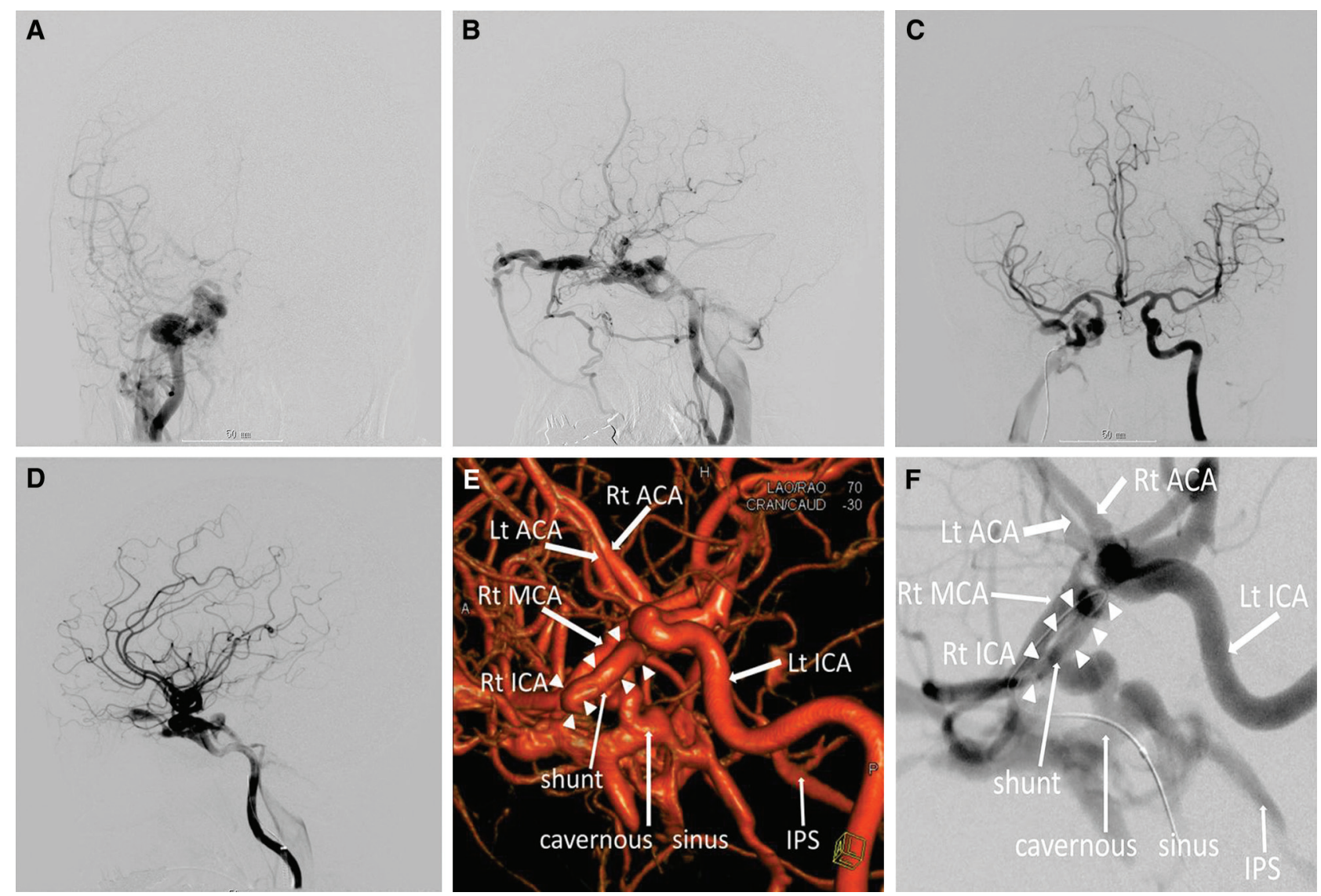

Fig. 1 (A and B) Preoperative angiography revealed a high flow shunt from the ICA to the cavernous sinus and remarkable intracranial venous reflux. The shunt has an extremely high flow; thus, it could not be identified on angiography of the right ICA (A: A-P view, B: lateral view). (C and D) Preoperative angiography revealed that the right ICA was occluded with a balloon catheter, and angiography was performed retrogradely from the left ICA to identify the shunt point (C: A-P view, D: lateral view). (E and F) Intraoperative working angle on angiography of the left internal carotid artery (E: 3D-digital subtraction angiography, F: left-front oblique view). IPS: inferior petrosal sinus, Lt ACA: right anterior cerebral artery, Lt ICA: left internal carotid artery, Rt ACA: right anterior cerebral artery, Rt ICA: right internal carotid artery (arrowhead).

(SOV). Moreover, magnetic resonance angiography (MRA) revealed a venous system, including the right CS. The patient was then diagnosed with traumatic DCCF and was referred to our hospital for treatment. Upon admission, she was alert and consciousness and had normal pupillary reflex and eye movement. The patient had bruit, exophthalmos, and bulbar conjunctival hyperemia in the right eye. However, no problems in the eyesight and field of vision and eye pressure were observed on ophthalmological examination.

Cerebral angiography revealed a shunt from the internal carotid artery (ICA) to the CS. However, no shunt was observed from the external carotid artery and vertebra-basilar arterial system, which indicated a Barrow type A CCF. The venous drainage routes back flowed to the SOV, and there was minimal blood flow via the intercavernous sinus to the contralateral
CS and intracranial venous reflux, such as reflux from the sphenoparietal sinus to the surficial sylvian vein, vein of Trolard, and SSS and from the uncal vein to the vein of Rosenthal. These findings indicated that the CCF could be treated. The shunt had an extremely high flow; thus, the shunt point could not be identified on angiography of the right ICA (Figs. 1A and 1B). In relation to this, the right ICA was occluded with a balloon catheter, and angiography was performed retrogradely from the left ICA to identify the shunt point (Figs. 1C and 1D).

Endovascular therapy: We planned to conduct transarterial and transvenous coil embolization with the transarterial balloon-assisted technique to prevent coil mass migration to the ICA if the fistula is not large. To prevent ischemic complications, $100 \mathrm{mg}$ of aspirin was administered 1 day before surgery. Under general anesthesia, a 7-Fr shuttle sheath 

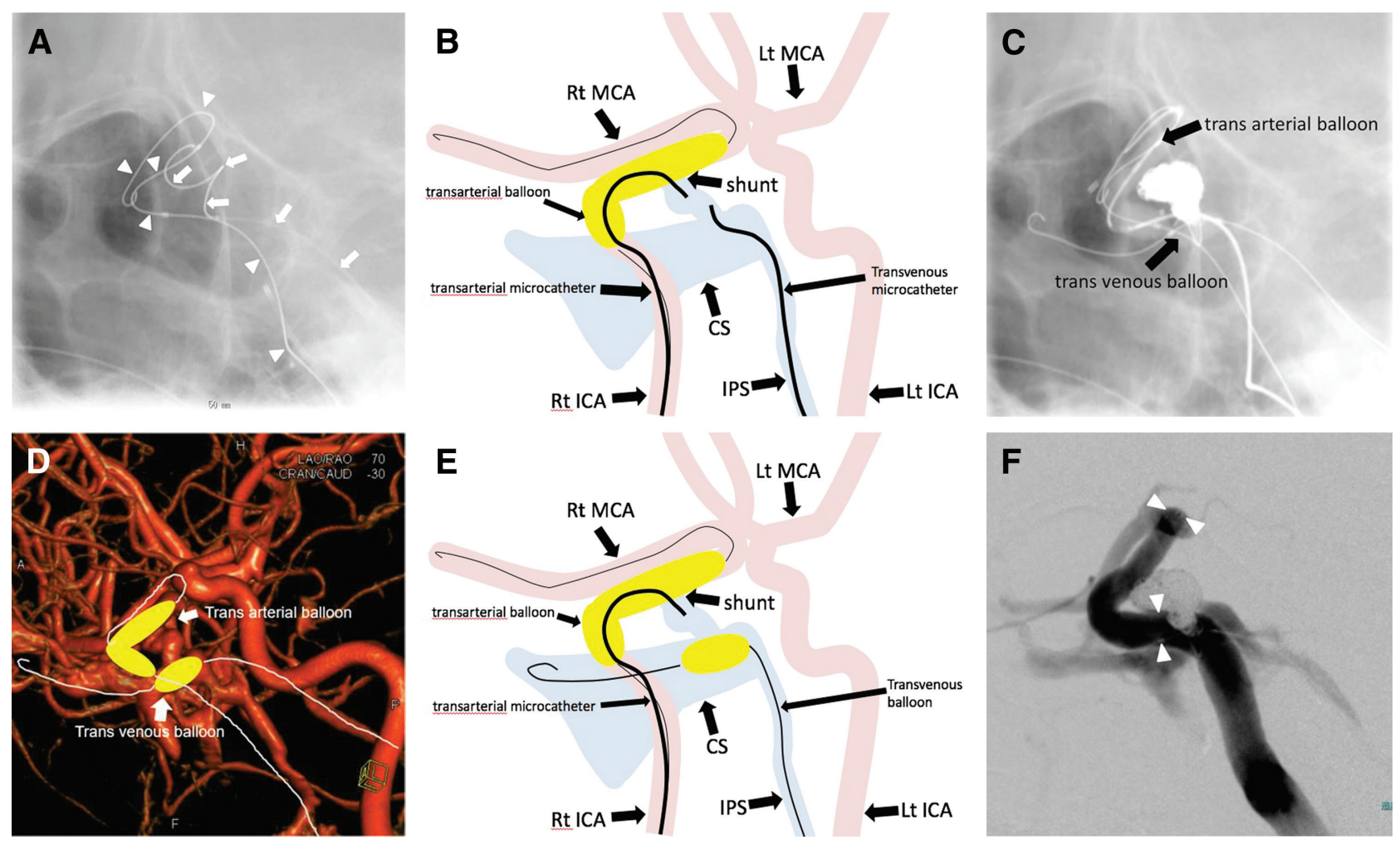

Fig. 2 (A) Transarterial balloon placement in the right ICA (arrowheads) and transvenous placement of a microcatheter to the cavernous sinus component of the vicinity of the fistula via the inferior petrosal sinus (arrows). (B) Schema of transarterial and transvenous coil embolization with transarterial balloon assisted technique. (C) Coil embolization using the combined transarterial and transvenous balloon-assisted technique. (D and E) Schema of coil embolization using the combined transarterial and transvenous balloon-assisted technique. (F) Enterprise VRD $4.5 \mathrm{~mm} / 25 \mathrm{~mm}$ was placed over the fistula to prevent migration of coils to the ICA. Arrow: proximal and distal marker of the stent. ICA: internal carotid artery.

(Cook Medical, Indianapolis, IN, USA) was inserted into the right femoral artery and was placed in the right ICA. A 6-Fr sheath was inserted into the left femoral vein and a 6-Fr Fubuki (Asahi Intecc Co., Ltd., Aichi, Japan) in the inferior petrosal sinus. A 4-Fr short sheath was inserted into the right brachial artery and a 4-Fr diagnostic catheter in the left ICA for angiography. The working angle was determined via a three-dimensional (3D) image analysis (Figs. $1 \mathrm{E}$ and $1 \mathrm{~F}$ ).

In total, 5000 units of heparin were administered intravenously. The transarterial placement of an SL-10 $90^{\circ}$ (Boston Scientific, Natick, MA, USA) to the shunt point with a 7-Fr shuttle sheath was performed using a Chikai Black 0.014 (Asahi Intecc Co., Ltd.). The transvenous placement of an SL-10 $45^{\circ}$ to the CS component of the vicinity of the fistula with a 6-Fr Fubuki was performed using a Chikai Black 0.014. To prevent migration of the coils to the ICA, HyperGlide $4 \mathrm{~mm} / 10 \mathrm{~mm}$ (Micro Therapeutics, Irvine, CA, USA) was placed in the ICA, and the Prowler select plus straight (Cordis
Neurovascular, Miami Lakes, FL, USA) was set in the middle cerebral artery using Chikai Black 0.014 for stent waits (Fig. 2A). The coil was inserted using the transarterial and transvenous microcatheter approaches with the transarterial balloon-assisted technique (Fig. 2B).

Because coils migrate easily to the frame outside, which is the dorsal component of the CS, and the tip of the SL-10 $45^{\circ}$ (transvenous microcatheter) was placed in the frame outside, HyperForm $4 \mathrm{~mm} /$ $7 \mathrm{~mm}$ (Micro Therapeutics) was used instead of the SL-10 $45^{\circ}$, and the balloon was inflated in the CS to perform combined transarterial and transvenous balloon-assisted coil embolization (Figs. 2C, 2D, and 2E). In all, 15 coils with a total length of $113 \mathrm{~cm}$ were used. Because finishing coils migrate easily to the ICA, Enterprise VRD $4.5 \mathrm{~mm} / 25 \mathrm{~mm}$ was placed over the fistula (Fig. 2F). Loading with $300 \mathrm{mg}$ of clopidogrel was administered immediately after surgery. Therapy with $100 \mathrm{mg} /$ day of aspirin and $75 \mathrm{mg} /$ day of clopidogrel was continuously provided. The shunt flow had decreased on the current 

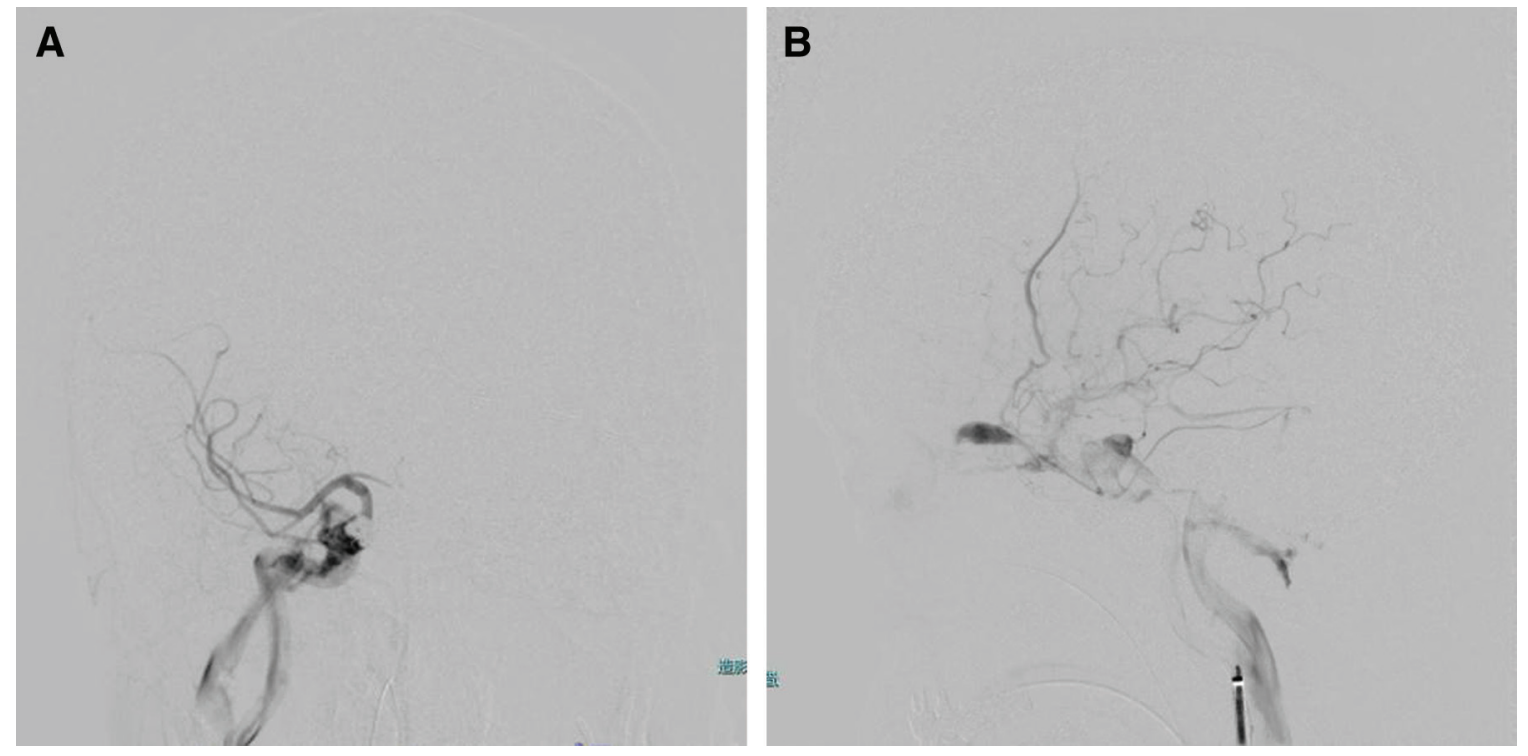

Fig. 3 Angiography performed immediately after surgery revealed the presence of the residues of a mild shunt, and intracranial venous reflux was observed on angiography of the right internal carotid artery (A: A-P view, B: lateral view).

angiography compared with the preoperative angiography. However, the shunt was still observed in the site. Angiography performed after heparin was reversed with protamine revealed the presence of a mild shunt (Fig. 3). Treatment was completed with the anticipation of thrombus formation with the disappearance of the shunt.

During the postoperative course, tinnitus and bruit disappeared immediately after surgery. Postoperative computed tomography scan revealed no apparent hemorrhagic complications. MRI on the day after surgery revealed the absence of complications, including acute-phase cerebral infarction, and improvement of SOV dilation. MRA revealed that the venous system disappeared. Moreover, exophthalmos and bulbar conjunctival hyperemia were not observed a few days after surgery. Angiography on the $7^{\text {th }}$ day after surgery revealed that the shunt had completely disappeared (Fig. 4). The patient did not present with neurological deficits and was discharged on postoperative day 12. The administration of clopidogrel as an antiplatelet drug was completed 6 months after surgery. Subsequently, only aspirin was continuously administered. No recurrence was observed 1 year after surgery. The patient provided informed consent for this case report.

\section{Discussion}

The first-line treatment for DCCF includes endovascular therapies, such as transarterial or transvenous embolization and embolization with stenting. The endovascular treatment for DCCF using a detachable balloon was first reported by Serbinenko et al. in 1974. ${ }^{12)}$

Meanwhile, Guglielmi et al. ${ }^{13)}$ have first described the treatment of DCCF using transvenous coil embolization in 1992. Since then, the outcomes of transarterial coil embolization have been excellent. ${ }^{2,4,14-16)}$ As a small fistula does not require several coils, coil embolization may be useful. In contrast, a large fistula needs more coils, and the coils may migrate to the parent artery, and patients are then at risk of long-term cranial neuropathy due to mass effect. Therefore, the technique may not be effective for a large fistula. ${ }^{1)}$ Another technique using Hydro coil has been proposed to reduce the number of coils. ${ }^{17)}$

Stent-assisted coil embolization has been performed to prevent migration of coils to the parent artery. ${ }^{3)}$ Morón et al. ${ }^{3)}$ have performed transarterial balloon and stent-assisted coil embolization in five patients with a DCCF and have achieved complete occlusion of the fistula in all cases. All patients underwent transvenous embolization using coils. However, two received NBCA intravenously during the last stage of treatment. In addition, the use of flow diverting stent for DCCF has been reported. ${ }^{18)}$

Gonzalez et al. ${ }^{1)}$ have reported about five patients treated using transarterial balloon-assisted embolization, including two who were diagnosed with DCCF. Among them, one underwent Onyx embolization and one Onyx and coil embolization. Occlusion of the fistula was achieved in both cases.

Some reports have shown the use of transarterial balloon-assisted coil embolization for the treatment 

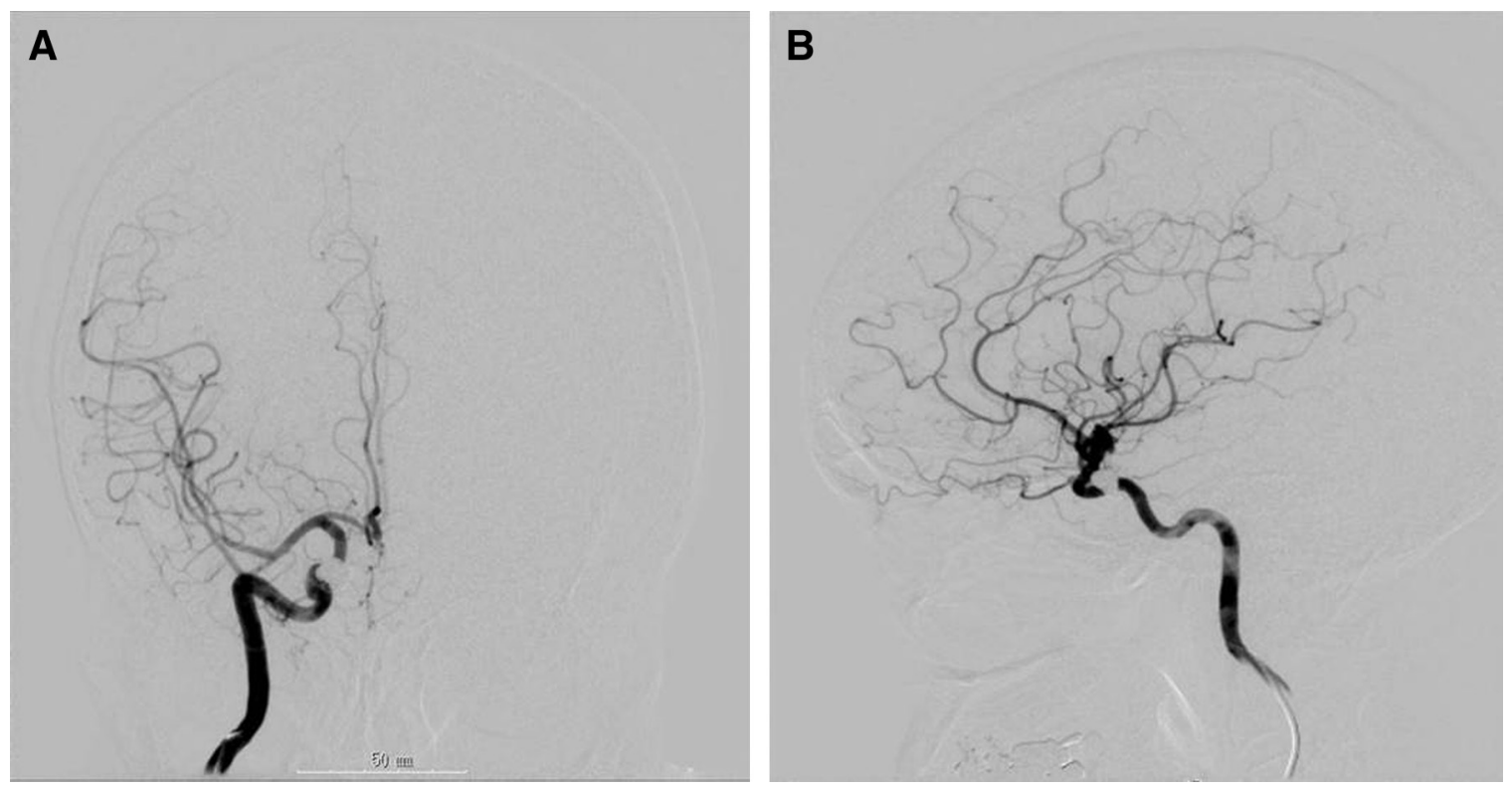

Fig. 4 On the 7th day after surgery, the shunt had completely disappeared on angiography of the right internal carotid artery (A: A-P view, B: lateral view).

of DCCF. However, transvenous balloon-assisted coil embolization has not been described previously. The transvenous balloon-assisted technique for the treatment of a DAVF using NBCA or Onyx has been used for flow control and prevention of migration to the adjacent blood vessels. ${ }^{7-11)}$ All patients underwent balloon-assisted embolization in the transverse sinus $^{7-9)}$ or SSS. ${ }^{10,11)}$ Only the patient in our case underwent balloon-assisted embolization in the CS.

The combined transarterial and transvenous balloon-assisted technique was used previously as an alternative for the plug of Onyx infusion for DAVF. ${ }^{4,10)}$ However, the purpose was different. In the present case, we used transarterial balloonassisted technique during coil embolization from arterial and both venous side for target embolization, because the fistula is not large in preoperative angiography. We performed coil embolization from the arterial and both venous sides, and a frame was established. However, finishing coils migrate easily to the frame outside. Therefore, we used the transvenous balloon-assisted technique and then the combined transarterial and transvenous balloonassisted technique at the middle. The migration of the coil to the frame outside was minimized, and the coil mass was compacted. Thus, target embolization was considered effective.

The case that the construction of a frame with only coil embolization using double catheter technique with both transarterial and transvenous microcatheters possibly exists. If coil migration to the frame outside is not considerable, without balloon-assisted embolization is continued for that case. However, it is thought that the transvenous balloon-assisted technique is useful when coil migration to the frame outside is considerable. Short-segment coil embolization using double balloon technique in an experimental vascular model has been reported, ${ }^{19)}$ and we performed double balloon technique with reference to it. Moreover, finishing coils migrate easily to the ICA; thus, transarterial balloon is slightly overinflated. However, the coil mass slightly moves when the balloon is deflated. Then, the stent is finally placed in the ICA.

The risks of using the balloon approach include bleeding due to injury in the venous sinus and cranial neuropathy particularly in the CS. In previous reports $^{7-11)}$ of transvenous balloon-assisted technique, high-compliance balloons, which are designed to assist with coil embolization of intracranial wideneck aneurysms, are preferred, but non-compliant balloons, such as Copernic (BaltExtrasion, Montmorency, France), have been used as their safety has been reported. ${ }^{7)}$ Caution must be taken when the balloon is inflated so as not to overinflate and rupture the sinus. To prevent this complication, an undersized balloon should be used, and caution is required to prevent overinflation. In our case, HyperGlide $4 \mathrm{~mm} / 10 \mathrm{~mm}$ was used based on the size of the fistula, and the postoperative outcome was excellent without complications.

If the fistula is not large, target embolization decreases the risk of cranial neuropathy caused by CS packing and reduces costs by decreasing the 
number of coils used, although the number and length of coils based on the sizes of the CS and fistula is difficult to compare. This technique can prevent CS packing using coil, and a normal venous flow can be maintained via the CS. This technique can prevent CS packing using a coil, and target embolization is then achieved and can reduce the number and length of the coils.

The case of transvenous coil embolization with transarterial balloon-assisted technique during target embolization for DCCF has been reported ${ }^{20)}$ However, coil embolization with double catheter technique from arterial and venous both sides during target embolization for DCCF has not been reported. In this case, the treatment in only double catheters was useful and safe; in fact, we performed the transvenous balloon-assisted technique for rescue because the filling coil migrates easily to the outside of the frame. Treatment in only double catheters may be possible using a frame made with different coil size.

\section{Conclusion}

In this case report, we described a successful treatment of a DCCF using the combined transarterial and transvenous balloon-assisted techniques, which promoted an effective target embolization. This report first showed that the balloon-assisted technique can be used for the treatment of fistula in the CS. The procedure has a risk of injury to the CS. However, no complications were observed in our case. Fewer coils are required using this approach; thus, we believe that the technique is effective for target embolization. Transvenous balloon-assisted technique in the CS was possible; however, safety was not established. Studies including a higher number of cases and safety examination are necessary in the future.

\section{Conflict of Interest Disclosure}

There are no conflicts of interest to declare.

\section{References}

1) Gonzalez LF, Chalouhi N, Tjoumakaris S, Jabbour P, Dumont AS, Rosenwasser HR: Treatment of carotid-cavernous fistulas using intraarterial balloon assistance: case series and technical note. Neurosurg Focus 35: E14, 2012

2) Luo CB, Teng MM, Chang FC, Lin CJ, Guo WY, Chang CY: Transarterial detachable coil embolization of direct carotid-cavernous fistula: immediate and long-term outcomes. J Chin Med Assoc 76: 31-36, 2013

3) Morón FE, Klucznik RP, Mawad ME, Strother CM: Endovascular treatment of high-flow carotid cavernous fistulas by stent-assisted coil placement. AJNR Am J Neuroradiol 26: 1399-1404, 2005

4) Chiu AH, Aw G, Wenderoth JD: Double-lumen arterial balloon catheter technique for Onyx embolization of dural arteriovenous fistulas: initial experience. J Neurointerv Surg 6: 400-403, 2014

5) Ertl L, Brückmann H, Kunz M, Crispin A, Fesl G: Endovascular therapy of low- and intermediategrade intracranial lateral dural arteriovenous fistulas: a detailed analysis of primary success rates, complication rates, and long-term follow-up of different technical approaches. J Neurosurg 126: 360-367, 2017

6) Shi ZS, Loh Y, Duckwiler GR, Jahan R, Viñuela F: Balloon-assisted transarterial embolization of intracranial dural arteriovenous fistulas. J Neurosurg 110: 921-928, 2009

7) Alturki AY, Enriquez-Marulanda A, Schmalz P, Ogilvy CS, Thomas AJ: Transarterial Onyx embolization of bilateral transverse-sigmoid dural arteriovenous malformation with transvenous balloon assist-initial U.S. experience with copernic RC venous remodeling balloon. World Neurosurg 109: 398-402, 2018

8) Jittapiromsak P, Ikka L, Benachour N, Spelle L, Moret J: Transvenous balloon-assisted transarterial Onyx embolization of transverse-sigmoid dural arteriovenous malformation. Neuroradiology 55: 345-350, 2013

9) Kerolus MG, Chung J, Munich SA, Matsuda Y, Okada H, Lopes DK: An Onyx tunnel: reconstructive transvervenous balloon-assisted Onyx embolization for dural arteriovenous fistula of the transverse-sigmoid sinus. J Neurosurg 129: 922-927, 2018

10) Piechowiak E, Zibold F, Dobrocky T, et al.: Endovascular treatment of dural arteriovenous fistulas of the transverse and sigmoid sinuses using transarterial balloon-assisted embolization combined with transvenous balloon protection of the venous sinus. AJNR Am J Neuroradiol 38: 1984-1989, 2017

11) Zhang Y, Li Q, Huang QH: Embolization of a superior sagittal sinus dural arteriovenous fistula under intrasinus balloon protection: a case report. Interv Neuroradiol 21: 94-100, 2015

12) Serbinenko FA: Balloon catheterization and occlusion of major cerebral vessels, J Neurosurg 41: 125-145, 1974

13) Guglielmi G, Viñuela F, Briganti F, Duckwiler G: Carotid-cavernous fistula caused by a ruptured intracavernous aneurysm: endovascular treatment by electrothrombosis with detachable coils. Neurosurgery 31: 591-596; discussion 596-597, 1992

14) Jansen O, Dörfler A, Forsting M, et al.: Endovascular therapy of arteriovenous fistulae with electrolytically detachable coils. Neuroradiology 41: 951-957, 1999

15) Mergeani A, Popescu D, Laza C, Dorobat B, Bajenaru OA, Antochi F: A review on endovascular techniques for treatment of direct post-traumatic carotid-cavernous fistula supported by case presentation. Maedica (Buchar) 7: 332-338, 2012 
16) Nesbit GM, Barnwell SL: The use of electrolytically detachable coils in treating high-flow arteriovenous fistulas. AJNR Am J Neuroradiol 19: 1565-1569, 1998

17) Wang ZG, Ding X, Zhang JQ, et al.: HydroCoil occlusion for treatment of traumatic carotid-cavernous fistula: preliminary experience. Eur J Radiol 71: 456-460, 2009

18) Nossek E, Zumofen D, Nelson E, et al.: Use of pipeline embolization devices for treatment of a direct carotid-cavernous fistula. Acta Neurochir (Wien) 157: 1125-1129; discussion 1130, 2015

19) Yunaiyama $D$, Saguchi $T$, Moriya $T$, et al.: Short-segment coil embolization using a double-balloon technique in an experimental vascular model. Cardiovasc Intervent Radiol 40: 1255-1260, 2017

20) Hayashi N, Okada H, Tomura N, et al.: Transvenous target embolization for a small sized, non-traumatic direct carotid-cavernous fistula using a single coil: technical case report. JNET J Neuroendovasc Ther 10: 272-277, 2016

Corresponding author: Ryotaro Suzuki, MD Department of Neurosurgery, Dokkyo Medical University Saitama Medical Center, 2-1-50 Minamikoshigaya, Koshigaya, Saitama 343-0855, Japan.

e-mail: ryo096943@gmail.com 